\title{
Author Correction: Genome-wide meta-analysis identifies new loci and functional pathways influencing Alzheimer's disease risk
}

Iris E. Jansen (D), Jeanne E. Savage (D), Kyoko Watanabe, Julien Bryois, Dylan M. Williams, Stacy Steinberg (D), Julia Sealock, Ida K. Karlsson, Sara Hägg DiD, Lavinia Athanasiu, Nicola Voyle, Petroula Proitsi D, Aree Witoelar, Sven Stringer (D, Dag Aarsland, Ina S. AlmdahI (D), Fred Andersen, Sverre Bergh, Francesco Bettella, Sigurbjorn Bjornsson, Anne Brækhus, Geir Bråthen (D), Christiaan de Leeuw DD, Rahul S. Desikan, Srdjan Djurovic (D), Logan Dumitrescu, Tormod Fladby, Timothy J. Hohman (D), Palmi V. Jonsson, Steven J. Kiddle (D), Arvid Rongve, Ingvild Saltvedt, Sigrid B. Sando, Geir Selbæk, Maryam Shoai, Nathan G. Skene D, Jon Snaedal, Eystein Stordal D, Ingun D. Ulstein, Yunpeng Wang, Linda R. White, John Hardy, Jens Hjerling-Leffler (D), Patrick F. Sullivan, Wiesje M. van der Flier, Richard Dobson (D), Lea K. Davis (D), Hreinn Stefansson, Kari Stefansson (D), Nancy L. Pedersen, Stephan Ripke, Ole A. Andreassen (D) and Danielle Posthuma (D)

Correction to: Nature Genetics https://doi.org/10.1038/s41588-018-0311-9, published online 7 January 2019.

In the version of this article initially published, in the phase 3 meta-analysis, the UK Biobank results were overweighed with a slightly larger (5\%) $N$, thus resulting in a minimal underestimation of effect sizes and in $P$ values marginally less significant than their actual values. Because the differences were on the order of the ninth decimal, there is no notable effect on the results or the conclusions drawn. The summary statistics have been updated accordingly at https://ctg.cncr.nl/software/summary_statistics. The Data availability statement has been updated to include the new URL in the HTML and PDF versions of the article.

Published online: 6 February 2020

https://doi.org/10.1038/s41588-019-0573-x

(c) The Author(s), under exclusive licence to Springer Nature America, Inc. 2020

\section{Author Correction: ARID1A influences HDAC1/BRD4 activity, intrinsic proliferative capacity and breast cancer treatment response}

Sankari Nagarajan (D), Shalini V. Rao, Joseph Sutton (D), Danya Cheeseman, Shanade Dunn, Evangelia K. Papachristou (D), Jose-Enrique Gonzalez Prada, Dominique-Laurent Couturier, Sanjeev Kumar, Kamal Kishore, Chandra Sekhar Reddy Chilamakuri, Silvia-Elena Glont, Emily Archer Goode, Cara Brodie, Naomi Guppy, Rachael Natrajan, Alejandra Bruna, Carlos Caldas (D), Alasdair Russell, Rasmus Siersbæk, Kosuke Yusa, lgor Chernukhin and Jason S. Carroll (D)

Correction to: Nature Genetics https://doi.org/10.1038/s41588-019-0541-5, published online 6 January 2020.

In the version of this article initially published, in Extended Data Fig. 6c,d, the graph key labels reading 'siARID1A' should have read 'siFOXA1'. The errors have been corrected in the HTML and PDF versions of the article.

Published online: 31 January 2020

https://doi.org/10.1038/s41588-020-0582-9

(c) The Author(s), under exclusive licence to Springer Nature America, Inc. 2020 